PROCEEDINGS OF THE

AMERICAN MATHEMATICAL SOCIETY

Volume 139, Number 10, October 2011, Pages 3633-3643

S 0002-9939(2011)10867-0

Article electronically published on February 24, 2011

\title{
THE WAT CONJECTURE ON THE TORUS
}

\author{
BASSAM SHAYYA
}

(Communicated by Michael T. Lacey)

\begin{abstract}
Let $\phi$ be a bounded holomorphic function on the unit disc $\mathbb{U} \subset \mathbb{C}$, let $k$ be an integer, and define the sequence $\left\{c_{n}\right\}$ by $c_{n}=\int_{\partial \mathbb{U}} \phi(z)^{n} z^{k-n} d z$. Nazarov and Shapiro conjectured that if $\|\phi\|_{L^{\infty}(\mathbb{U})} \leq 1$ and $\phi$ is not a rotation, then $\lim _{n \rightarrow \infty} c_{n}=0$. A consequence of this conjecture would be that any composition operator is weakly asymptotically Toeplitz on the Hardy space $H^{2}(\mathbb{U})$. We formulate a higher-dimensional version of this conjecture and use Fourier analytic techniques to obtain results that improve on what is currently known in dimension one.
\end{abstract}

\section{INTRODUCTION}

Let $\mathbb{U}$ be the open unit disc in the complex plane $\mathbb{C}$ and let $H(\mathbb{U})$ be the space of all holomorphic functions on $\mathbb{U}$. Let $\phi \in H(\mathbb{U})$ be such that $\phi$ is not a rotation 1 and $|\phi(z)|<1$ for all $z \in \mathbb{U}$. For $h \in H(\mathbb{U})$, define

$$
\mathcal{C}_{\phi} h=h \circ \phi .
$$

By Littlewood's subordination principle, the linear operator $\mathcal{C}_{\phi}: H(\mathbb{U}) \rightarrow H(\mathbb{U})$ restricts to a bounded linear operator on the Hardy space $H^{2}(\mathbb{U})$, and hence we can associate with $\mathcal{C}_{\phi}$ an infinite matrix whose columns are the coefficients of the Maclaurin series expansions of the functions $\mathcal{C}_{\phi} z^{\alpha}=\phi^{\alpha}, \alpha=0,1,2, \ldots$ So if $c_{\alpha, \beta}$ is the entry in the $\alpha$ th column and $\beta$ th row of the matrix associated with $\mathcal{C}_{\phi}$, then 2

$$
c_{\alpha, \beta}=\int_{\partial \mathbb{U}} \phi(z)^{\alpha} z^{-\beta} \frac{d z}{2 \pi i z} .
$$

Let $S$ be the forward shift operator on $H^{2}(\mathbb{U})$ given by

$$
S z^{\alpha}=z^{\alpha+1} \quad(\alpha=0,1,2, \ldots) .
$$

Then the adjoint $S^{*}$ of $S$ is the backward shift operator on $H^{2}(\mathbb{U})$ given by

$$
S^{*} z^{\alpha}= \begin{cases}z^{\alpha-1} & \text { if } \alpha>0 \\ 0 & \text { if } \alpha=0\end{cases}
$$

The composition operator $\mathcal{C}_{\phi}$ is said to be weakly asymptotically Toeplitz if the sequence $\left\{S^{* n} \mathcal{C}_{\phi} S^{n}\right\}$ of bounded linear operators on $H^{2}(\mathbb{U})$ converges in the weak

Received by the editors June 21, 2010 and, in revised form, September 1, 2010

2010 Mathematics Subject Classification. Primary 42B05; Secondary 47 B33.

${ }^{1}$ A rotation in this paper is a function $\mathbb{U} \rightarrow \mathbb{U}$ given by $z \mapsto a z$ with $|a|=1$.

${ }^{2}$ The function $\phi$ initially defined on $\mathbb{U}$ can be extended to be defined almost everywhere on $\partial \mathbb{U}$ by defining $\phi\left(e^{i \theta}\right)=\lim _{r \rightarrow 1^{-}} \phi\left(r e^{i \theta}\right)$. 
operator topology. Equivalently, $\mathcal{C}_{\phi}$ is weakly asymptotically Toeplitz if

$$
\lim _{n \rightarrow \infty} c_{n, n-k}=0
$$

for all $k \in \mathbb{Z}$. It is easy to see that if $\phi(0)=0$, then $\mathcal{C}_{\phi}$ is weakly asymptotically Toeplitz (see [6]). The WAT conjecture, as formulated by Nazarov and Shapiro in [6], asserts that the weak asymptotic Toeplitzness of $\mathcal{C}_{\phi}$ continues to hold when $\phi(0) \neq 0$. In a related paper, 8 , Shapiro showed that the sequence $\left\{c_{n, n-k}\right\}$ converges to zero in the Cesàro $(C, \alpha)$ sense for any $\alpha>0$ (the case $\alpha=1$ had been established in 6]).

Let $E=\{z \in \partial \mathbb{U}:|\phi(z)|=1\}$ and write

$$
c_{n, n-k}=a_{n, n-k}+b_{n, n-k}
$$

with

$$
a_{n, n-k}=\int_{\partial \mathbb{U} \backslash E} \phi(z)^{n} z^{k-n} \frac{d z}{2 \pi i z} \quad \text { and } \quad b_{n, n-k}=\int_{E} \phi(z)^{n} z^{k-n} \frac{d z}{2 \pi i z}
$$

for $n \in \mathbb{N}$ and $k \in \mathbb{Z}$. Clearly, $\lim _{n \rightarrow \infty} a_{n, n-k}=0$, so the WAT conjecture has the following equivalent formulation.

The WAT conjecture on the circle $([6])$. Suppose $\phi \in H(\mathbb{U}),|\phi(z)| \leq 1$ for all $z \in \mathbb{U}$, and $\phi(0) \neq 0$. Then

$$
\lim _{n \rightarrow \infty} b_{n, n-k}=0
$$

for all $k \in \mathbb{Z}$.

It is currently not known if (11) holds even when $k=0$. The best previously known result about the $b_{n, n-k}$ is

$$
\lim _{N \rightarrow \infty} \frac{1}{N} \sum_{n=1}^{N}\left|b_{n, n-k}\right|^{2}=0
$$

for all $k \in \mathbb{Z}$, as was shown in [6]. One of the highlights of the present paper is a quantitative strengthening of (2), in the case $k=0$, to

$$
\frac{1}{N} \sum_{n=M}^{M+N}\left|b_{n, n}\right|^{2} \leq \frac{C}{\log N}
$$

for all $M \geq 1$ and $N \geq 2$, where $C$ is a constant that depends only on $\phi(0)$. For general $k \in \mathbb{Z}$, we obtain the following improvement on (2):

$$
\sum_{n=1}^{\infty} \frac{\left|b_{n, n-k}\right|^{2}}{n}<\infty
$$

The proof that (4) implies (2) is an easy summation by parts argument (see [1]). There are also results similar to (4) obtained in [1] for $c_{n, n-k}$ instead of $b_{n, n-k}$ using complex analytic methods and only for $k \geq 0$.

The fact that $\phi$ is holomorphic in the unit disc implies - via the theorem of F. and M. Riesz - that the function $\phi\left(e^{i \theta}\right)^{n}$ cannot remain constant on a set of positive arc-length measure on the circle, and hence it oscillates and the oscillations become faster as $n$ gets larger, and so one expects $b_{n, n-k}$ to become small as $n$ becomes large. To prove (3) and (4) then, one has to find the right technology to translate the smallness of the sets $\left\{e^{i \theta} \in E: \phi\left(e^{i \theta}\right)=c\right\},|c|=1$, into decay estimates on $b_{n, n-k}$. Before we try to make this more precise, though, we would like to shift 
our perspective from complex to Fourier analysis. Therefore, we shall think of the circle as the interval $\mathbb{T}=[-1 / 2,1 / 2]$ with the understanding that a function (or measure) on $\mathbb{T}$ is a one-periodic function (or measure) on $\mathbb{R}$, and instead of a function $\phi \in H(\mathbb{U})$ bounded by one, we shall deal with a function $f$ on $\mathbb{T}$ such that $\|f\|_{L^{\infty}(\mathbb{T})} \leq 1$ and $\widehat{f}(n)=0$ for all negative integers $n$, and instead of the condition $\phi(0) \neq 0$, we shall impose the condition $\widehat{f}(0) \neq 0$. In this context,

$$
c_{n, n-k}=\int_{-1 / 2}^{1 / 2} f(x)^{n} e^{-2 \pi i n x} e^{2 \pi i k x} d x
$$

and

$$
b_{n, n-k}=\int_{E} f(x)^{n} e^{-2 \pi i n x} e^{2 \pi i k x} d x,
$$

where $E=\{x \in \mathbb{T}:|f(x)|=1\}$. Now

$$
\begin{aligned}
\frac{1}{N} \sum_{n=1}^{N} b_{n, n-k} & =\int_{E} \frac{e^{2 \pi i k x}}{N} \sum_{n=1}^{N}\left(f(x) e^{-2 \pi i x}\right)^{n} d x \\
& =\int_{E} \frac{f(x) e^{2 \pi i k x}}{N} \frac{1-\left(f(x) e^{-2 \pi i x}\right)^{N}}{F(x)} d x
\end{aligned}
$$

where $F(x)=e^{2 \pi i x}-f(x)$. The integrand is bounded by one and converges to zero as $N \rightarrow \infty$ at each point $x \in E$ where $F(x) \neq 0$, and hence at almost every point in $E$. Applying Lebesgue's dominated convergence theorem, we conclude that

$$
\lim _{N \rightarrow \infty} \frac{1}{N} \sum_{n=1}^{N} b_{n, n-k}=0
$$

for all $k \in \mathbb{Z}$. The fact that $F(x) \neq 0$ for almost every $x \in E$ follows from the following celebrated theorem of F. and M. Riesz applied to the measure $d \lambda(x)=$ $F(x) d x$.

The theorem of F. and M. Riesz. Suppose $\lambda \in M(\mathbb{T}),\|\lambda\|>0$, and $\widehat{\lambda}(n)=0$ for all $n<0$. Then $d \lambda$ and $d x$ have the same null sets:

$$
\int_{A} d|\lambda|=0 \Leftrightarrow \int_{A} d x=0 .
$$

For a very nice discussion on the theorem of F. and M. Riesz, we refer the reader to [4. A crucial observation is that to now upgrade 3 from (5) to (2), one needs to play the same game with

$$
\left|b_{n, n-k}\right|^{2}=\int_{E} \int_{E} f(x)^{n} \overline{f(y)^{n}} e^{-2 \pi i n(x-y)} e^{2 \pi i k(x-y)} d x d y,
$$

and hence needs a higher-dimensional version of the theorem of F. and M. Riesz to guarantee that the set $\{(x, y) \in E \times E: F(x, y)=0\}$ has Lebesgue measure zero, where now $F(x, y)=e^{2 \pi i(1,-1) \cdot(x, y)}-f(x) \overline{f(y)}$. Fortunately, this is provided by the following theorem of Bochner [2] and de Leeuw and Glicksberg [3] (see also [5]).

Theorem A ([2] and [3]). Suppose $\lambda \in M\left(\mathbb{T}^{d}\right),\|\lambda\|>0$, and $\hat{\lambda}(\xi)=0$ for all lattice points $\xi$ in the interior of a cone with opening greater than $\pi$. Then $d \lambda$ and $d x$ have the same null sets.

\footnotetext{
${ }^{3}$ The authors of [6] proceed from (5) to (2) by showing that $b_{n, n-k}$ are the Fourier coefficients of a continuous measure on the circle and then using Wiener's mass point theorem.
} 
To prove (4), we shall need to obtain more precise information about the set $\{(x, y) \in E \times E: F(x, y)=0\}$. This will be provided to us by Helson's and Lowdenslager's remarkable generalization of Szegö's theorem to higher dimensions, to which we now turn our attention.

Following Helson and Lowdenslager [5], we say that a set $S \subset \mathbb{Z}^{d}$ is a half-space of lattice points in $\mathbb{R}^{d}$ if

(i) $0 \notin S$,

(ii) $\xi \neq 0$ implies $\xi \in S$ or $-\xi \in S$,

(iii) $\xi, \xi^{\prime} \in S$ implies $\xi+\xi^{\prime} \in S$.

Notice that if $S$ is a half-space of lattice points in $\mathbb{R}^{d}$, then so is $-S=\{-\xi: \xi \in S\}$. Also, if $S_{1}$ and $S_{2}$ are half-spaces of lattice points in $\mathbb{R}^{d_{1}}$ and $\mathbb{R}^{d_{2}}$, respectively, then $S_{1} \times \mathbb{R}^{d_{2}} \cup \mathbb{R}^{d_{1}} \times S_{2}$ contains a half-space of lattice points in $\mathbb{R}^{d_{1}} \times \mathbb{R}^{d_{2}}$ (e.g., $\left.S_{1} \times \mathbb{R}^{d_{2}} \cup\{0\} \times S_{2}\right)$.

Theorem B ([5]). Suppose $w$ is a nonnegative function in $L^{1}\left(\mathbb{T}^{d}\right)$ and $S$ is a half-space of lattice points in $\mathbb{R}^{d}$. Then

$$
e^{\int_{\mathbb{T}^{d}} \log w(x) d x}=\inf \int_{\mathbb{T}^{d}}|1+P(x)|^{2} w(x) d x,
$$

where the infimum is taken over all trigonometric polynomials on $\mathbb{R}^{d}$ of the form $P(x)=\sum_{\xi \in S} a_{\xi} e^{2 \pi i \xi \cdot x}$.

We are going to apply Theorem $\mathrm{B}$ in the following form.

Corollary A ([5]). Suppose $f \in L^{2}\left(\mathbb{T}^{d}\right), S$ is a half-space of lattice points in $\mathbb{R}^{d}$, and $\widehat{f}(\xi)=0$ for all $\xi \in S$. Then

$$
\int_{\mathbb{T}^{d}} \log |f(x)| d x \geq \log |\widehat{f}(0)| .
$$

Proof ([5]). By Theorem B (applied with $w=|f|^{2}$ ),

$$
e^{\int_{\mathbb{T}^{d}} \log |f(x)|^{2} d x}=\inf \int_{\mathbb{T}^{d}}|1+P(x)|^{2}|f(x)|^{2} d x,
$$

where the infimum is taken over all trigonometric polynomials on $\mathbb{R}^{d}$ of the form $P(x)=\sum_{\xi \in S} a_{\xi} e^{2 \pi i \xi \cdot x}$. The integral on the left-hand side of the above equality does not change if we replace $P$ by $\bar{P}$, and, by Plancherel's theorem,

$$
\int_{\mathbb{T}^{d}}|1+\overline{P(x)}|^{2}|f(x)|^{2} d x \geq|\widehat{h}(0)|^{2},
$$

where $h=(1+\bar{P}) f$. Since

$$
\widehat{h}(0)=\widehat{f}(0)+\sum_{\xi \in S} \overline{a_{\xi}} \widehat{f}(\xi)=\widehat{f}(0)
$$

it follows that

$$
e^{\int_{\mathbb{T}^{d}} \log |f(x)|^{2} d x} \geq|\widehat{f}(0)|^{2}
$$

as desired.

Finally, to establish (3), we shall show that $\left|b_{n, n}\right|^{2}$ are the Fourier coefficients of a positive measure $\mu \in M(\mathbb{T})$. Then (4) becomes

$$
\sum_{n=1}^{\infty} \frac{\widehat{\mu}(n)}{n}<\infty
$$


The convergence of the above series combined with the fact that $\mu$ is a positive measure with nonnegative Fourier coefficients plays a crucial role in our argument.

Having outlined our method of proof in the one-dimensional case, when $f$ is a function on the circle $\mathbb{T}$, we remind the reader that all the results of this paper will be established for functions defined on the $d$-torus $\mathbb{T}^{d}$. These results are stated in the following theorem.

Theorem 1. Suppose $f \in L^{\infty}\left(\mathbb{T}^{d}\right)$ with $\|f\|_{L^{\infty}\left(\mathbb{T}^{d}\right)} \leq 1, g \in L^{1}\left(\mathbb{T}^{d}\right)$, and $\nu \in \mathbb{Z}^{d}$. For $n \in \mathbb{N}$, define

$$
c_{n}=\int_{\mathbb{T}^{d}} f(x)^{n} e^{-2 \pi i n \nu \cdot x} g(x) d x .
$$

(i) If $\widehat{f}(\xi)=0$ for all $\xi$ in a half-space $S$ of lattice points and $\nu \in-S$, then 4

$$
\sum_{n=1}^{\infty} \frac{\left|c_{n}\right|^{2}}{n} \leq\left(\log \frac{e^{4}}{|\widehat{f}(0)|^{2}}\right)\|g\|_{L^{\infty}\left(\mathbb{T}^{d}\right)}^{2}+\frac{\pi}{2}\|g\|_{L^{1}\left(\mathbb{T}^{d}\right)}^{2} .
$$

(ii) If $\widehat{f}(\xi)=0$ for all $\xi$ in a half-space $S$ of lattice points, $\nu \in-S$, and $g$ is real-valued, then

$$
\sum_{n=1}^{\infty} \frac{\left|c_{n}\right|^{2}}{n} \leq\left(\log \frac{e^{4}}{|\widehat{f}(0)|^{2}}\right)\|g\|_{L^{\infty}\left(\mathbb{T}^{d}\right)}^{2} .
$$

(iii) If $\widehat{f}(\xi)=0$ for all $\xi$ in a half-space $S$ of lattice points, $\nu \in-S$, and $g$ is identically equal to one, then

$$
\sum_{n=1}^{\infty} \frac{\left|c_{n}\right|^{2}}{n} \leq \log \frac{1}{|\widehat{f}(0)|^{2}}
$$

(iv) If $\widehat{f}(\xi)=0$ for all $\xi \in \mathbb{Z}^{d}$ such that $\xi \cdot u \leq c$ for some unit vector $u \in \mathbb{R}^{d}$ and some constant $c \in \mathbb{R}$, then

$$
\lim _{N \rightarrow \infty} \frac{\left|c_{1}\right|^{2}+\cdots+\left|c_{N}\right|^{2}}{N}=0 .
$$

(v) If $\widehat{f}(\xi)=0$ for all $\xi$ in a half-space $S$ of lattice points, $\nu \in-S, \widehat{f}(0) \neq 0$, and $g$ is the characteristic function of any measurable subset of $\left\{x \in \mathbb{T}^{d}:|f(x)|=1\right\}$, then

$$
\frac{1}{N} \sum_{n=M}^{M+N}\left|c_{n}\right|^{2} \leq \frac{C}{\log N}
$$

for all $M \geq 1$ and $N \geq 2$, where $C$ is a constant that depends only on $|\widehat{f}(0)|$.

Among other things, part (v) of Theorem 1 tells us that

$$
\min _{M \leq n \leq M+N}\left|c_{n}\right| \leq\left(\frac{C}{\log N}\right)^{1 / 2}
$$

for all $M \geq 1$ and $N \geq 2$. Also by (v), if $\phi \in H(\mathbb{U})$ is an inner function with $\phi(0) \neq 0$, then

$$
\frac{1}{N} \sum_{n=M}^{M+N}\left|\int_{E}\left(\frac{\phi(z)}{z}\right)^{n} \frac{d z}{2 \pi i z}\right|^{2} \leq \frac{C}{\log N}
$$

\footnotetext{
${ }^{4}$ The case $\widehat{f}(0)=0$ is covered by the convention $\log \frac{1}{0}=-\log 0=\infty$. So if either $\widehat{f}(0)=0$ or $\|g\|_{L^{\infty}\left(\mathbb{T}^{d}\right)}=\infty$, then the right-hand side of the inequality in (i) is $\infty$ and the inequality is automatically satisfied. Similarly for the inequalities in (ii) and (iii).
} 
for all $M \geq 1, N \geq 2$, and any measurable $E \subset \partial \mathbb{U}$.

Motivated by Theorem 1 and the discussion preceding it, we find it reasonable to conjecture the following higher dimensional version of the WAT conjecture.

The WAT conjecture on the torus. Suppose $f \in L^{\infty}\left(\mathbb{T}^{d}\right), S$ is a half-space of lattice points in $\mathbb{R}^{d},\|f\|_{L^{\infty}\left(\mathbb{T}^{d}\right)} \leq 1, \widehat{f}(\xi)=0$ for all $\xi \in S, \nu \in-S$, and $\widehat{f}(0) \neq 0$. Then

$$
\lim _{n \rightarrow \infty} \int_{\left\{x \in \mathbb{T}^{d}:|f(x)|=1\right\}} f(x)^{n} e^{-2 \pi i(n-k) \nu \cdot x} d x=0
$$

for all $k \in \mathbb{Z}$.

\section{Proof of Theorem 1}

We start by writing

$$
\left|c_{n}\right|^{2}=\int_{\mathbb{T}^{d}} \int_{\mathbb{T}^{d}}(f(x) \overline{f(y)})^{n} e^{-2 \pi i n \nu \cdot(x-y)} g(x) \overline{g(y)} d x d y
$$

and using the assumption $\|f\|_{L^{\infty}\left(\mathbb{T}^{d}\right)} \leq 1$ to see that

$$
\begin{aligned}
\sum_{n=1}^{\infty} \frac{r^{n}\left|c_{n}\right|^{2}}{n} & =\int_{\mathbb{T}^{d}} \int_{\mathbb{T}^{d}} g(x) \overline{g(y)} \sum_{n=1}^{\infty} \frac{r^{n}}{n}\left(f(x) \overline{f(y)} e^{-2 \pi i \nu \cdot(x-y)}\right)^{n} d x d y \\
& =-\int_{\mathbb{T}^{d}} \int_{\mathbb{T}^{d}} g(x) \overline{g(y)} \log \left(1-\frac{r f(x) \overline{f(y)}}{e^{2 \pi i \nu \cdot(x-y)}}\right) d x d y \\
& =-\int_{\mathbb{T}^{d}} \int_{\mathbb{T}^{d}} g(x) \overline{g(y)} \log \frac{F(x, y)}{e^{2 \pi i \nu \cdot(x-y)}} d x d y
\end{aligned}
$$

for all $0<r<1$, where $F: \mathbb{T}^{d} \times \mathbb{T}^{d} \rightarrow \mathbb{C}$ is given by

$$
F(x, y)=e^{2 \pi i(\nu,-\nu) \cdot(x, y)}-r f(x) \overline{f(y)} .
$$

Notice that $\widehat{F}(\xi, \eta)=0$ for all $(\xi, \eta) \in S \times \mathbb{R}^{d} \cup \mathbb{R}^{d} \times(-S)$, and hence for all $(\xi, \eta)$ in a half-space of lattice points in $\mathbb{R}^{d} \times \mathbb{R}^{d}$, so it follows by Corollary $\mathrm{A}$ that

$$
\int_{\mathbb{T}^{d}} \int_{\mathbb{T}^{d}} \log |F(x, y)| d x d y \geq \log |\widehat{F}(0,0)| .
$$

Since $\sum_{n=1}^{\infty} r^{n}\left|c_{n}\right|^{2} / n$ is nonnegative, (6) becomes

$$
\begin{aligned}
\sum_{n=1}^{\infty} \frac{r^{n}\left|c_{n}\right|^{2}}{n} & =\left|\int_{\mathbb{T}^{d}} \int_{\mathbb{T}^{d}} g(x) \overline{g(y)} \log \frac{F(x, y)}{e^{2 \pi i \nu \cdot(x-y)}} d x d y\right| \\
& \leq \int_{\mathbb{T}^{d}} \int_{\mathbb{T}^{d}}|g(x) g(y)|\left|\log \frac{F(x, y)}{e^{2 \pi i \nu \cdot(x-y)}}\right| d x d y \\
& \leq \int_{\mathbb{T}^{d}} \int_{\mathbb{T}^{d}}|g(x) g(y)|\left((\log |F(x, y)|)^{2}+\frac{\pi^{2}}{4}\right)^{1 / 2} d x d y \\
& \leq\|g\|_{L^{\infty}\left(\mathbb{T}^{d}\right)}^{2} \int_{\mathbb{T}^{d}} \int_{\mathbb{T}^{d}}|\log | F(x, y)|| d x d y+\frac{\pi}{2}\|g\|_{L^{1}\left(\mathbb{T}^{d}\right)}^{2} .
\end{aligned}
$$


Now

$$
\begin{aligned}
\int_{\mathbb{T}^{d}} & \int_{\mathbb{T}^{d}}|\log | F(x, y)|| d x d y \\
\quad & =\iint_{X}|\log | F(x, y)|| d x d y+\iint_{X^{c}}|\log | F(x, y)|| d x d y \\
& =\iint_{X} \log |F(x, y)| d x d y-\iint_{X^{c}} \log |F(x, y)| d x d y,
\end{aligned}
$$

where $X=\left\{(x, y) \in \mathbb{T}^{d} \times \mathbb{T}^{d}:|F(x, y)| \geq 1\right\}$, and (by (77)

$$
\iint_{X} \log |F(x, y)| d x d y+\iint_{X^{c}} \log |F(x, y)| d x d y \geq \log |\widehat{F}(0,0)|,
$$

so

$$
\begin{aligned}
\int_{\mathbb{T}^{d}} \int_{\mathbb{T}^{d}}|\log | F(x, y)|| d x d y & \leq 2 \iint_{X} \log |F(x, y)| d x d y+\log \frac{1}{|\widehat{F}(0,0)|} \\
& \leq 2 \iint_{X}|F(x, y)| d x d y+\log \frac{1}{r|\widehat{f}(0)|^{2}} \\
& \leq 2\|F\|_{L^{\infty}\left(\mathbb{T}^{d}\right)}+\log \frac{1}{r|\widehat{f}(0)|^{2}} \\
& \leq 2+2 r+\log \frac{1}{r}+\log \frac{1}{|\widehat{f}(0)|^{2}},
\end{aligned}
$$

and so

$$
\sum_{n=1}^{\infty} \frac{r^{n}\left|c_{n}\right|^{2}}{n} \leq\|g\|_{L^{\infty}\left(\mathbb{T}^{d}\right)}^{2}\left(2+2 r+\log \frac{1}{r}+\log \frac{1}{|\widehat{f}(0)|^{2}}\right)+\frac{\pi}{2}\|g\|_{L^{1}\left(\mathbb{T}^{d}\right)}^{2} .
$$

Letting $r \rightarrow 1$, we obtain (i).

When $g$ is real-valued, (6) tells us that

$$
\begin{aligned}
\sum_{n=1}^{\infty} \frac{r^{n}\left|c_{n}\right|^{2}}{n} & =-\int_{\mathbb{T}^{d}} \int_{\mathbb{T}^{d}} g(x) g(y) \log |F(x, y)| d x d y \\
& \leq\|g\|_{L^{\infty}\left(\mathbb{T}^{d}\right)}^{2} \int_{\mathbb{T}^{d}} \int_{\mathbb{T}^{d}}|\log | F(x, y)|| d x d y \\
& \leq\|g\|_{L^{\infty}\left(\mathbb{T}^{d}\right)}^{2}\left(2+2 r+\log \frac{1}{r}+\log \frac{1}{|\widehat{f}(0)|^{2}}\right)
\end{aligned}
$$

and (ii) follows by letting $r \rightarrow 1$.

When $g$ is identically equal to one, we can obtain a better estimate by maneuvering a little differently. Starting with ([6), we now have

$$
\sum_{n=1}^{\infty} \frac{r^{n}\left|c_{n}\right|^{2}}{n}=-\int_{\mathbb{T}^{d}} \int_{\mathbb{T}^{d}} \log \frac{F(x, y)}{e^{2 \pi i \nu \cdot(x-y)}} d x d y .
$$

Since $\sum_{n=1}^{\infty} r^{n}\left|c_{n}\right|^{2} / n$ is real, this becomes

$$
\sum_{n=1}^{\infty} \frac{r^{n}\left|c_{n}\right|^{2}}{n}=-\int_{\mathbb{T}^{d}} \int_{\mathbb{T}^{d}} \log |F(x, y)| d x d y,
$$


and hence by (7),

$$
\sum_{n=1}^{\infty} \frac{r^{n}\left|c_{n}\right|^{2}}{n} \leq \log \frac{1}{|\widehat{F}(0,0)|}=\log \frac{1}{r|\widehat{f}(0)|^{2}} .
$$

Letting $r \rightarrow 1$, we obtain (iii).

For $(x, y) \in \mathbb{T}^{d} \times \mathbb{T}^{d}$, define

$$
G(x, y)=e^{2 \pi i(\nu,-\nu) \cdot(x, y)}-f(x) \overline{f(y)}
$$

and let

$$
Y=\left\{(x, y) \in \mathbb{T}^{d} \times \mathbb{T}^{d}: G(x, y)=0\right\} .
$$

Then $\widehat{G}(\xi, \eta)=0$ for all $(\xi, \eta) \in \mathbb{Z}^{d} \times \mathbb{Z}^{d}$ such that

$$
\xi \cdot u \leq c_{1} \quad \text { and } \quad \eta \cdot u \geq c_{2}
$$

for some constants $c_{1}, c_{2} \in \mathbb{R}$. So by Theorem $\mathrm{A}$ (applied to the measure $d \lambda(x, y)=$ $G(x, y) d x d y)$, the set $Y$ has measure zero, and so

$$
\left|c_{n}\right|^{2}=\iint_{Y^{c}}(f(x) \overline{f(y)})^{n} e^{-2 \pi i n \nu \cdot(x-y)} g(x) \overline{g(y)} d x d y .
$$

Thus

$$
\begin{aligned}
\frac{1}{N} \sum_{n=1}^{N}\left|c_{n}\right|^{2} & =\iint_{Y^{c}} \frac{g(x) \overline{g(y)}}{N} \sum_{n=1}^{N}\left(f(x) \overline{f(y)} e^{-2 \pi i \nu \cdot(x-y)}\right)^{n} d x d y \\
& =\iint_{Y^{c}} \frac{f(x) g(x) \overline{f(y) g(y)}}{N} \frac{1-\left(f(x) \overline{f(y)} e^{-2 \pi i \nu \cdot(x-y)}\right)^{N}}{G(x, y)} d x d y \\
& \rightarrow 0 \text { as } N \rightarrow \infty,
\end{aligned}
$$

where we have employed the $L^{\infty}$ bound on $f$ and the $L^{1}$ bound on $g$ to apply Lebesgue's dominated convergence theorem.

The rest of this section concerns the proof of (v). Write $g=\chi_{E}$ with $E \subset\{x \in$ $\left.\mathbb{R}^{d}:|f(x)|=1\right\}$. For $n \in \mathbb{Z}$, define

$$
\alpha_{n}=\int_{E} \int_{E}(f(x) \overline{f(y)})^{n} e^{-2 \pi i n \nu \cdot(x-y)} d x d y,
$$

so that $\alpha_{-n}=\alpha_{n}=\left|c_{n}\right|^{2}$ for $n \geq 1$. Then

$$
\begin{aligned}
\sum_{j=1}^{m} & \sum_{k=1}^{m} z_{j} \overline{z_{k}} \alpha_{j-k} \\
& =\int_{E} \int_{E} \sum_{j=1}^{m} \sum_{k=1}^{m} z_{j}\left(f(x) \overline{f(y)} e^{-2 \pi i \nu \cdot(x-y)}\right)^{j} \overline{z_{k}\left(f(x) \overline{f(y)} e^{-2 \pi i \nu \cdot(x-y)}\right)^{k}} d x d y \\
& =\int_{E} \int_{E}\left|\sum_{j=1}^{m} z_{j}\left(f(x) \overline{f(y)} e^{-2 \pi i \nu \cdot(x-y)}\right)^{j}\right|^{2} d x d y \\
& \geq 0
\end{aligned}
$$

for any complex constants $z_{1}, \ldots, z_{m}$, and it follows by Herglotz's theorem that there is a positive Borel measure on $\mathbb{T}$ such that $\widehat{\mu}(n)=\alpha_{n}$ for all $n \in \mathbb{Z}$. Part (ii) 
tells us that

On the other hand 5

$$
\sum_{n=1}^{\infty} \frac{\widehat{\mu}(n)}{n} \leq \log \frac{e^{4}}{|\widehat{f}(0)|^{2}}
$$

$$
\int_{-1 / 2}^{1 / 2} \log \frac{1}{1-r e^{-2 \pi i \theta}} d \mu(\theta)=\int_{-1 / 2}^{1 / 2} \sum_{n=1}^{\infty} \frac{r^{n} e^{-2 \pi i n \theta}}{n} d \mu(\theta)=\sum_{n=1}^{\infty} r^{n} \frac{\widehat{\mu}(n)}{n},
$$

so that

$$
\int_{-1 / 2}^{1 / 2} \log \frac{1}{\left|e^{2 \pi i \theta}-r\right|} d \mu(\theta)=\sum_{n=1}^{\infty} r^{n} \frac{\widehat{\mu}(n)}{n} \leq \log \frac{e^{4}}{|\widehat{f}(0)|^{2}}
$$

for all $0 \leq r<1$. Since

$$
\left|e^{2 \pi i \theta}-r\right| \leq\left|e^{2 \pi i \theta}-1\right|+1-r \leq 2 \pi|\theta|+1-r,
$$

it follows that

$$
\int_{-1 / 2}^{1 / 2} \log \frac{1}{2 \pi|\theta|+1-r} d \mu(\theta) \leq \log \frac{e^{4}}{|\widehat{f}(0)|^{2}}
$$

i.e.

$$
\begin{aligned}
& \int_{-r /(2 \pi)}^{r /(2 \pi)} \log \frac{1}{2 \pi|\theta|+1-r} d \mu(\theta) \\
& \quad \leq \log \frac{e^{4}}{|\widehat{f}(0)|^{2}}+\int_{r /(2 \pi) \leq|\theta| \leq 1 / 2} \log (2 \pi|\theta|+1-r) d \mu(\theta) \\
& \quad \leq \log \frac{e^{4}}{|\widehat{f}(0)|^{2}}+\|\mu\| \log (\pi+1-r) \\
& \quad=\log \frac{e^{4}}{|\widehat{f}(0)|^{2}}+|E|^{2} \log (\pi+1-r) .
\end{aligned}
$$

In the last line we used the fact that $\|\mu\|=\widehat{\mu}(0)=|E|^{2}$, where $|E|$ denotes the Lebesgue measure of $E$. Letting $r \rightarrow 1$ and using Fatou's lemma, we conclude that

$$
\int_{-1 /(2 \pi)}^{1 /(2 \pi)} \log \frac{1}{2 \pi|\theta|} d \mu(\theta) \leq \log \frac{e^{4}}{|\widehat{f}(0)|^{2}}+|E|^{2} \log \pi \leq \log \frac{\pi e^{4}}{|\widehat{f}(0)|^{2}}
$$

Thus

$$
\int_{-\epsilon}^{\epsilon} d \mu \leq \frac{C_{1}}{\log \frac{1}{2 \pi \epsilon}}
$$

whenever $0<\epsilon \leq 1 /(2 \pi)$, where

$$
C_{1}=\log \frac{\pi e^{4}}{|\widehat{f}(0)|^{2}} .
$$

To turn (8) into an estimate on the averages of the Fourier coefficients of $\mu$, we proceed as follows.

Let $\psi$ be a $C_{0}^{\infty}$ function on $\mathbb{R}$ such that $\operatorname{supp} \psi$ is contained in the interval $\left(-\frac{1}{2}, \frac{1}{2}\right), \widehat{\psi}$ is nonnegative, and $\widehat{\psi} \geq 1$ on the interval $(-1,1)$. For $N=1,2, \ldots$, set

$$
\psi_{N}(t)=e^{2 \pi i M t} \psi(N t)
$$

\footnotetext{
${ }^{5}$ By part (iv) and Wiener's mass point theorem, $\mu(\{x\})=0$ for all $x \in \mathbb{T}$, so there is no confusion in writing $\int_{-1 / 2}^{1 / 2}$ for $\int_{\mathbb{T}}$ when we integrate against $\mu$.
} 
for $t \in \mathbb{R}$. Then $\operatorname{supp} \psi_{N}$ is contained in the interval $\left(-\frac{1}{2 N}, \frac{1}{2 N}\right)$ and

$$
\widehat{\psi_{N}}(s)=\frac{1}{N} \widehat{\psi}\left(\frac{s-M}{N}\right) \geq \frac{1}{N}
$$

for all $s \in(M-N, M+N)$. This gives

$$
\frac{\left|c_{M}\right|^{2}+\cdots+\left|c_{M+N}\right|^{2}}{N} \leq \sum_{n=M-N}^{M+N} \frac{\widehat{\mu}(n)}{N} \leq \sum_{n=M-N}^{M+N} \widehat{\psi_{N}}(n) \widehat{\mu}(n) \leq \sum_{n=-\infty}^{\infty} \widehat{\psi_{N}}(n) \widehat{\mu}(n) .
$$

On the other hand,

$$
\begin{aligned}
\sum_{n=-\infty}^{\infty} \widehat{\psi_{N}}(n) \widehat{\mu}(n) & =\sum_{n=-\infty}^{\infty} \widehat{\psi_{N}}(n) \int_{-1 / 2}^{1 / 2} e^{-2 \pi i n \theta} d \mu(\theta) \\
& =\int_{-1 / 2}^{1 / 2} \sum_{n=-\infty}^{\infty}\left(\widehat{\psi_{N}}(n) e^{-2 \pi i n \theta}\right) d \mu(\theta)
\end{aligned}
$$

where the interchange of sum and integral is justified by the Schwartz decay of $\widehat{\psi_{N}}$. Also, extending $\psi_{N}$ to a one-periodic function on $\mathbb{R}$, we obtain a function in $C_{0}^{\infty}(\mathbb{T})$ with the Fourier series

$$
\sum_{n=-\infty}^{\infty} \widehat{\psi_{N}}(n) e^{2 \pi i n \theta}
$$

and it follows by Fourier inversion on the circle that

$$
\sum_{n=-\infty}^{\infty} \widehat{\psi_{N}}(n) e^{-2 \pi i n \theta}=\psi_{N}(-\theta)
$$

for all $-1 / 2 \leq \theta \leq 1 / 2$. Thus

$$
\begin{aligned}
\frac{\left|c_{M}\right|^{2}+\cdots+\left|c_{M+N}\right|^{2}}{N} & \leq \int_{-1 / 2}^{1 / 2} \psi_{N}(-\theta) d \mu(\theta) \\
& =\int_{-1 /(2 N)}^{1 /(2 N)} \psi_{N}(-\theta) d \mu(\theta) \\
& \leq\|\psi\|_{L^{\infty}(\mathbb{R})} \int_{-1 /(2 N)}^{1 /(2 N)} d \mu \\
& \leq \frac{C_{1}\|\psi\|_{L^{\infty}(\mathbb{R})}}{\log \frac{N}{\pi}}
\end{aligned}
$$

provided $N \geq \pi$, where in the last line we have used (8).

We would like to conclude with a remark about (8). The analysis leading to this inequality can be easily modified to give

$$
\mu\left(I_{\epsilon}\right) \leq \frac{C_{1}}{\log \frac{1}{2 \pi \epsilon}}
$$

for any interval $I_{\epsilon}$ of radius $0<\epsilon \leq 1 /(2 \pi)$. (For $\theta_{0} \in \mathbb{R}$ and $0 \leq r<1$, we have

$$
\begin{aligned}
\int_{\theta_{0}-1 / 2}^{\theta_{0}+1 / 2} \log \frac{1}{1-r e^{-2 \pi i\left(\theta-\theta_{0}\right)}} d \mu(\theta) & =\int_{\theta_{0}-1 / 2}^{\theta_{0}+1 / 2} \sum_{n=1}^{\infty} \frac{r^{n} e^{-2 \pi i n\left(\theta-\theta_{0}\right)}}{n} d \mu(\theta) \\
& =\sum_{n=1}^{\infty} r^{n} e^{2 \pi i n \theta_{0}} \frac{\widehat{\mu}(n)}{n}
\end{aligned}
$$


$\mathrm{SO}$

$$
\begin{aligned}
\int_{\theta_{0}-1 / 2}^{\theta_{0}+1 / 2} \log \frac{1}{\left|e^{2 \pi i\left(\theta-\theta_{0}\right)}-r\right|} d \mu(\theta) & =\sum_{n=1}^{\infty} r^{n} \cos \left(2 \pi n \theta_{0}\right) \frac{\widehat{\mu}(n)}{n} \\
& \leq \sum_{n=1}^{\infty} \frac{\widehat{\mu}(n)}{n} \\
& \leq \log \frac{e^{4}}{|\widehat{f}(0)|^{2}} .
\end{aligned}
$$

Proceeding as before, we obtain

$$
\int_{\theta_{0}-\epsilon}^{\theta_{0}+\epsilon} d \mu \leq \frac{C_{1}}{\log \frac{1}{2 \pi \epsilon}}
$$

for all $0<\epsilon \leq 1 /(2 \pi)$ and $\theta_{0} \in \mathbb{R}$.) This is an estimate on the modulus of continuity of $\mu$, which, in particular, implies that $\mu$ is absolutely continuous with respect to the Hausdorff measure $H$ on $\mathbb{T}$ with determining function (e.g. see [7])

$$
h(t)=\frac{1}{\log \frac{1}{\pi t}} \quad(0 \leq t \leq 1 / \pi) .
$$

\section{REFERENCES}

[1] F. Abi-Khuzam and B. Shayya, A remark on the WAT conjecture, preprint, 2009.

[2] S. Bochner, Boundary values of analytic functions in several variables and of almost periodic functions, Ann. of Math. 45 (1944), 708-722. MR0011132 (6:124a)

[3] K. DE Leeuw AND I. Glicksberg, Quasi-invariance and analyticity of measures on compact groups, Acta Math. 109 (1963), 179-205. MR0144152 (26:1700)

[4] F. Forelli, The theorem of F. and M. Riesz for unbounded measures, The Madison Symposium on Complex Analysis (Madison, WI, 1991), 221-234, Contemp. Math., 137, Amer. Math. Soc., Providence, RI, 1992. MR.1190984 (94f:28014)

[5] H. Helson and D. Lowdenslager, Prediction theory and Fourier series in several variables, Acta Math. 99 (1958), 165-202. MR0097688 (20:4155)

[6] F. Nazarov and J. Shapiro, On the Toeplitzness of composition operators, Complex Var. Elliptic Equ. 52 (2007), 193-210. MR2297770 (2008a:47043)

[7] J. Shapiro, Hausdorff measure and Carleson thin sets, Proc. Amer. Math. Soc. 79 (1980), 67-71. MR560586 (81m:28001)

[8] J. Shapiro, Every composition operator is (mean) asymptotically Toeplitz, J. Math. Anal. Appl. 333 (2007), 523-529. MR2323505 (2008d:47050)

Department of Mathematics, American University of Beirut, Beirut, Lebanon

E-mail address: bshayya@aub.edu.lb 